OSCAR HUERTA

ESCUELA DE DISEÑO

Y CENTRO DE ENERGÍA UC

FACULTAD DE ARQUITECTURA,

DISEÑO Y ESTUDIOS URBANOS

PONTIFICIA UNIVERSIDAD CATÓLICA DE CHILE, SANTIAGO, CHILE.

OHUERTA@UC.CL
Fecha de recepción: 13/08/2020

Fecha de aceptación: 23/10/2020

Cómo citar: Huerta, O. (2020).

Ecodiseño de envases para una

economía circular.

RChD: creación y pensamiento, 5(9), 1-12.

https//doi.org/10.5354/0719-837X.2020.58303

Revista Chilena de Diseño,

RChD: creación y pensamiento

Universidad de Chile

2020, 5(9).

http://rchd.uchile.cl

\section{Ecodiseño de envases para una economía circular}

\author{
Packaging Ecodesign for a Circular Economy
}

Resumen. Envases y embalajes cumplen funciones importantes para la protección y manejo de productos, antes de llegar a sus destinatarios y durante su uso o consumo. Sus residuos post consumo, sin embargo, impactan negativamente al medio ambiente. Por esto en Chile están afectos a la ley de responsabilidad extendida del productor, en su avance hacia una economía circular. Los impactos de los envases y embalajes provienen, no solo de sus residuos, sino de todo su ciclo de vida. Este artículo se orienta a una audiencia de profesionales y académicos de Diseño y tiene dos objetivos: indagar sobre las mejores formas de implementar una economía circular para minimizar los impactos negativos de ciclo de vida de envases y embalajes, e indagar sobre los roles que las disciplinas de Diseño pueden desempeñar en el contexto de la ley. Entre los elementos de la ley, el ecodiseño emerge como un instrumento especialmente relevante para evitar impactos negativos. Su ámbito de acción, desde la planificación, Diseño y desarrollo de productos y envases, es estratégico para la toma de decisiones con responsabilidad ambiental ayudando a prevenir sus impactos. Las disciplinas de Diseño suelen participar en etapas tempranas del desarrollo de productos y envases. Esta posición privilegiada les otorga el potencial de ser parte instrumental de las soluciones en envases y embalajes para el cumplimiento de la ley minimizando impactos ambientales.

Palabras clave: Ciclo de vida, ecodiseño, envases y embalajes, impacto ambiental, termodinámica.

\begin{abstract}
Packaging fulfills important functions for the protection and handling of products, before reaching their recipients and during their use or consumption. Its post-consumer waste, however, negatively impacts the environment. For this reason, in Chile packaging is subject to the extended producer responsibility law, as the country advances towards a circular economy. The impacts of containers and packaging come, not only from their waste, but from their entire life cycle. This article is aimed at an audience of Design professionals and academics and has two objectives: to investigate the best ways to implement a circular economy in order to minimize packaging life cycle negative impacts, and to inquire about the roles that Design disciplines can play in the context of the extended producer responsibility law. Among the elements of the law, ecodesign emerges as a particularly relevant instrument to help avoiding negative impacts. Its scope of action, from the planning, design and development of products and packaging, is strategic for making environmentally responsible decisions and aid in preventing negative impacts. Design disciplines are often involved in the early stages of product and packaging development. This privileged position gives them the potential to be an instrumental part of solutions in packaging ecodesign for compliance with the law while minimizing environmental impacts.
\end{abstract}

Keywords: Ecodesign, environmental impact, packaging, product life cycle, thermodynamics. 


\section{Introducción.}

\section{Próximas obligaciones ambientales para los envases y embalajes}

El término en inglés packaging denomina a envases y embalajes, e incluye envases que contienen productos y embalajes que reúnen productos envasados para su transporte y manejo durante la distribución. Los envases y embalajes son ángeles y demonios en el sistema productivo y de consumo contemporáneo. Por una parte, cumplen múltiples funciones como objeto de uso, tales como contención, transporte o preservación, entre otras; y como objeto de sentido, creador e instaurador de significación (Ciravegna, 2017). Por otra parte, los envases y embalajes globalmente constituyen millones de toneladas mensuales de residuos que provocan impactos negativos en el medio ambiente, ocupan espacio en rellenos sanitarios o vertederos y generan costos ambientales y financieros.

Debido a estos problemas han surgido internacionalmente leyes de responsabilidad extendida del productor. Junto a estas leyes se han establecido sistemas de gestión de residuos como parte importante de la solución para su cumplimiento. Ejemplos son el sistema Ecoembes en España, Der Grüne Punkt alemán (también llamado Green Dot), Fost Plus en Bélgica y Stewardship Ontario en Canadá. La experiencia internacional, de unos treinta años, ha logrado aumentar significativamente las cantidades de residuos que se valorizan y disminuir las que se disponen en rellenos sanitarios. También han servido como antecedentes para el avance de nuestro país en materias de gestión de residuos sólidos hacia una economía circular.

En Chile la recientemente publicada Ley 20.920 Marco Para la Gestión de Residuos, la Responsabilidad Extendida del Productor y Fomento al Reciclaje (REP) obligará al productor de los productos afectos a ella (productos prioritarios) a organizar y financiar la gestión de sus residuos al final de su vida útil (Ministerio del Medio Ambiente de Chile [MMA], 2016). Los productos prioritarios son problemáticos como residuos post consumo y causan impactos negativos en el medio ambiente. Se establecieron cinco criterios con los que un producto debe cumplir para ser prioritario: consumo masivo, volumen significativo, residuos peligrosos, factibilidad de valorización y regulación comparada (MMA, 2016).

Los envases y embalajes son productos prioritarios y cumplen plenamente con cuatro de los cinco criterios, especialmente consumo masivo y volumen significativo. Cabe mencionar que en el caso de envases y embalajes será obligatorio para el productor de los productos envasados organizar y financiar la gestión de los residuos de los envases utilizados, y no realmente el productor, como sucede con los otros cinco productos prioritarios. Una vez que la REP esté en pleno funcionamiento se espera un aumento en la proporción de residuos valorizados, que formarán parte de nuevos productos o serán fuente de energía.

El Ministerio del Medio Ambiente de Chile ha implementado la Oficina de Economía Circular para reducir el impacto ambiental de los residuos y promover un modelo de economía circular (MMA, 2020). Sus dos ámbitos principales de acción son la implementación de la REP, y catalizar un entorno de innovación, marco regulatorio y otros instrumentos para avanzar 
hacia una economía circular (MMA, 2020). La REP constituye un cambio de paradigma para la gestión de residuos en Chile (Aylwin \& Bortnick, 2017).

Impacto ambiental de ciclo de vida de envases y embalajes

Las grandes cantidades de residuos de envases post consumo que generamos impactan negativamente al medio ambiente. Los impactos de envases y embalajes, sin embargo, no provienen solamente de sus residuos. Para comprender los impactos de un producto es necesaria una aproximación más amplia enfocada en su ciclo de vida completo (Büsser $\&$ Jungbluth, 2009). Desde una perspectiva de ciclo de vida, los envases y embalajes son productos entendidos como sistemas. Esto sirve para analizar y para proyectar, o Diseñar.

El ciclo de vida de envases y embalajes comienza con la etapa de extracción de materias primas. Éstas se utilizan para la producción de materiales e insumos. Luego viene la manufactura, en que se producen envases y embalajes. Prosigue la etapa de distribución, en que los envases se transportan hacia centros de distribución, puntos de venta o productores de productos a envasar. A continuación, se envasan productos y se utilizan embalajes que agrupan varias unidades de productos envasados para su transporte y manejo durante la distribución. Eventualmente los productos envasados llegan a puntos de venta minorista en donde los consumidores los adquieren. Posteriormente el consumidor utiliza los envases durante tiempos variables, según el caso. Finalmente, el consumidor descarta el envase cuando ya no le es útil y comienza la etapa denominada fin de vida, cuando los residuos se reutilizan, reciclan, incineran o se disponen en rellenos sanitarios. Una fracción de envases y embalajes son retornables y como tales no son residuos, sino más bien vuelven a sus dueños para ser limpiados y acondicionados para envasar o embalar nuevos productos.

Durante las etapas del ciclo de vida de un producto se utilizan procesos en que se intercambia materia y energía con el medio ambiente, y se provocan impactos negativos (Wenzel, Hauschild \& Alting, 1997). Esto aplica a envases y embalajes en su calidad de productos. Los procesos requieren materiales y energía para funcionar; luego directa o indirectamente generan emisiones de gases, efluentes líquidos y residuos sólidos, con resultado de impacto ambiental.

En la primera etapa del ciclo de vida de envases y embalajes ocurre la extracción de materias primas. Habitualmente su obtención y la manufactura de productos provocan significativos impactos ambientales (Cabeza, Rincón, Vilariño, Pérez \& Castell, 2014). La manufactura utiliza energía y otros recursos. Luego, la distribución de productos hasta los puntos de venta o sus destinatarios finales provoca impactos por transporte, alimentado principalmente por diésel. El manejo de carga en bodegas y centros de distribución se realiza con vehículos y máquinas que utilizan energía. En los puntos de venta se generan residuos de embalajes. Tras la venta de los productos los usuarios muchas veces generan casi inmediatamente residuos de envases. En otros casos el envase dura más tiempo en uso, pero finalmente es descartado como residuo. Un porcentaje menor de estos residuos se reutiliza o se recicla. En menor cantidad también sucede que el usuario le da un segundo uso al envase, el cual puede durar años en su nueva 
función (e.g., envase de latón como costurero). En Chile a la mayor parte de los residuos de envases y embalajes se les da disposición final. En algunos casos se usan como combustible alternativo.

\section{Ventajas y desventajas del reciclaje}

En Chile el reciclaje de envases ha existido desde hace décadas. Se reciclan algunos materiales, en lo cual intervienen recolectores, empresas que compran residuos para revender o para procesarlos y manufacturar productos nuevos. El reciclaje se ha dado de manera espontánea y de acuerdo con el funcionamiento del mercado. Se prevé que con la implementación de la REP habrá un incremento importante del reciclaje y otras formas de valorización.

El reciclaje tiene dos ventajas potenciales, una ambiental y otra económica. La primera es que los residuos a reciclar ya no terminarán en rellenos sanitarios ni serán dispuestos de otro modo, y así evita los problemas que esto trae. La segunda es que los materiales en los residuos pasan de no tener valor y constituir una responsabilidad para sus dueños, a formar parte de productos con valor añadido y que tienen lugar en el mercado.

El problema es que el reciclaje equivale a un procesamiento significativo de los residuos, para fabricar con ellos productos nuevos. Esto conlleva el uso de energía e insumos, que dependen de los procesos empleados, y la generación de residuos y emisiones varias. Además, los productos reciclados no están hechos cien por ciento de residuos, sino que incorporan material virgen para mantener un nivel de calidad adecuado a su uso. Adicionalmente, en muchos casos los residuos recorren largas distancias desde donde se generan hasta donde se reciclan, y así generan impactos por transporte. En otras palabras, el reciclaje en su conjunto también provoca impacto ambiental.

\section{Disposición final de residuos}

En Chile existe disposición final legal e ilegal de residuos sólidos, entre éstos los envases y embalajes. Parte de estos residuos se acumulan ilegalmente en vertederos o son quemados al aire libre. En cuanto a la disposición legal de residuos, la mayor parte se dispone en rellenos sanitarios, otras cantidades se usan como combustible alternativo. Ambas opciones legales generan impactos negativos en el medioambiente. En el caso de los rellenos sanitarios, el tratamiento de residuos en condiciones anaeróbicas crea una nueva corriente de residuos y emisiones problemáticas para el medio ambiente (Kiser, 2016). En el caso de la incineración, esta opción para el tratamiento de residuos genera más gases de efecto invernadero que el relleno sanitario (Dormer, Finn, Ward \& Cullen, 2013).

\section{Planteamiento del problema y preguntas de investigación}

Por una parte, se impulsa en Chile una economía circular cuyo principal motor es la REP. Por otra parte, el cumplimiento de la REP, si bien en parte evita problemas ambientales derivados de residuos de envases, también genera nuevos impactos debido a los procesos, uso de recursos y emisiones que provienen de la valorización de los residuos. Estos impactos se suman a los que provienen de las demás etapas del ciclo de vida de envases y embalajes. 
Este problema lleva a la siguiente pregunta de investigación: entre las opciones para el cumplimiento de la REP ¿de qué maneras la REP y la economía circular se pueden implementar para un mejor resultado que minimice el impacto ambiental de ciclo de vida de envases y embalajes? De forma complementaria ¿qué roles podrían cumplir las disciplinas de Diseño para apoyar el cumplimiento de la REP y minimizar estos impactos?

\section{Metodología.}

\section{Objetivo e hipótesis}

El trabajo tiene dos objetivos principales. El primero es indagar acerca de las maneras de cumplir con la REP que pueden posibilitar una minimización de los impactos ambientales negativos en el ciclo de vida de envases y embalajes. El segundo objetivo es descubrir qué roles pueden desempeñar las disciplinas de Diseño en el contexto de la REP de envases y embalajes.

La hipótesis planteada es que las disciplinas de Diseño pueden tener un rol relevante para el cumplimiento de la REP de manera tal que los impactos de ciclo de vida de envases y embalajes se puedan minimizar.

\section{Métodos}

Se realizó una revisión de literatura para sentar las bases del artículo con respecto a cuatro temas. El primero es la REP chilena y antecedentes internacionales de leyes similares, junto a los sistemas de gestión de residuos que facilitan su cumplimiento. El segundo tema es el ciclo de vida de los envases y embalajes y sus impactos ambientales. El tercer tema es el reciclaje de residuos de envases, en cuanto a sus aspectos positivos y externalidades negativas. El cuarto tema es la disposición final de residuos que no han sido reciclados ni valorizados de otra manera. Se consultaron artículos científicos, documentos gubernamentales, normas internacionales y otras fuentes de literatura académica.

La revisión de literatura permitió identificar el problema de que los envases y embalajes provocan impacto ambiental en todas las etapas de su ciclo de vida y no solamente cuando se generan como residuos. De manera similar, la implementación de la REP y la valorización de residuos, si bien reduce en parte los impactos por residuos, tiene también como resultado otros impactos ambientales atribuibles a los envases y embalajes, y que se suman a sus demás impactos de ciclo de vida. Junto a lo anterior, la revisión de literatura permitió establecer preguntas de investigación e hipótesis.

A fin de confirmar o refutar la hipótesis y responder las preguntas de investigación se plantea el análisis de tres elementos: la Ley 20.920 por una parte, el concepto de economía circular por otra, y teorías sobre ecodiseño y otras aproximaciones relevantes para minimizar impactos negativos de productos.

\section{Resultados}

La REP contiene más de treinta definiciones relevantes para sus efectos. Entre estas definiciones se encuentran dos conceptos claves para cumplir la ley y lograr sus objetivos de disminuir la generación de residuos, fomentar su valorización y proteger la salud de las personas y el medio ambiente: el primero es ciclo de vida del producto y el segundo es ecodiseño. Ambos 
términos provienen de las áreas de ingeniería, desarrollo industrial y Diseño de productos, que han incorporado conocimiento de ciencias biológicas y ecología para proponer estrategias para la creación de productos con mínimo impacto.

\section{Ciclo de vida del producto}

La definición de ciclo de vida del producto que se encuentra en la ley corresponde a la definición en la norma ISO 14.044: el ciclo de vida de un producto se ha definido como las etapas consecutivas e interrelacionadas de un sistema producto, desde la obtención de sus materias primas hasta su disposición final $($ ISO, 2006). El producto se entiende como bienes o servicios (ISO,2006).

La perspectiva de ciclo de vida puede aplicarse tanto para la evaluación de impactos ambientales de bienes y servicios (productos), como para el Diseño o rediseño de productos y sistemas con el objetivo de minimizar sus impactos negativos.

Dentro de la evaluación de impactos, existe la evaluación de ciclo de vida (LCA por su sigla en inglés). Ésta es una técnica principalmente cuantitativa pero también cualitativa para evaluar el impacto ambiental de un sistema productivo. Existen también las técnicas denominadas análisis de costos de ciclo de vida (LCC) y evaluación social de ciclo de vida (SLCA), cuyos resultados son un importante complemento para los resultados de LCA a fin de conocer de manera integral el desempeño de sostenibilidad de bienes y servicios.

\section{Ecodiseño}

Ecodiseño, en tanto, es una aproximación para el Diseño y desarrollo de productos que busca llevar al mínimo sus impactos ambientales de ciclo de vida. El ecodiseño procura integrar conocimiento de ciclo de vida tempranamente en el desarrollo de un producto para lograr su óptimo desempeño en términos económicos y ecológicos (Karlsson \& Luttropp, 2006).

El origen del ecodiseño se remonta a los años ochenta en Delft University of Technology (TU Delft), Países Bajos, como resultado del proyecto PROMISE que buscaba implementar la creación de productos de bajo impacto ambiental, como estrategia para levantar la industria nacional holandesa (Sterrenberg, Böttcher \& Hoo, 1997). Posteriormente el término ecodiseño nace para el resto del mundo gracias a la publicación en inglés del manual Ecodesign: a promising approach for sustainable production and consumption (Huerta, 2014). En esta publicación se presenta un diagrama denominado Rueda de estrategias de ecodiseño que contiene ocho estrategias para mejorar el desempeño ambiental de un producto. Cada estrategia contiene un número de acciones específicas para aplicar.

Posteriormente se desarrolló una nueva versión de la rueda de estrategias de ecodiseño en Estados Unidos, basada en la de Tu Delft, la cual incluye estrategias de Diseño para: innovación, reducción de impactos por materiales, innovación en la manufactura, distribución eficiente, reducción de impactos en uso y comportamiento de usuarios, longevidad del sistema, sistemas de transición y optimización de fin de vida (White, St. Pierre \& Belletire, 2013). 
Por su parte, la Agencia Federal Alemana de Medio Ambiente (UBA) ha definido ecodiseño así:

El término ecodiseño significa desarrollo y Diseño de productos con conciencia ambiental. Describe una manera sistemática que tiene como objetivo incluir los aspectos ambientales en el proceso de planificación, desarrollo y Diseño del producto lo antes posible. Esto significa que el 'medio ambiente' se agrega como un criterio de desarrollo de productos junto con otros criterios clásicos de funcionalidad, rentabilidad, seguridad, confiabilidad, ergonomía, factibilidad técnica y, por último, pero no menos importante, estética. (Tischner, Schmincke, Rubik, F.\& Prosler, 2000, p. 12).

En la definición se entiende que el ecodiseño no es muy diferente del Diseño convencional, excepto porque incorpora consideraciones sobre el medio ambiente durante el Diseño y desarrollo de los productos. Mientras antes se incorpore el ecodiseño, mayor será su alcance para reducir el impacto ambiental de un producto (Tischner et al., 2000).

\section{Principios relevantes en la REP}

Once principios inspiran la REP. Dos de estos principios pueden ser especialmente relevantes para minimizar impactos ambientales a través de la planificación, Diseño y desarrollo de productos.

El primero es el principio de jerarquía en el manejo de residuos. Éste presenta un orden de preferencia para el manejo de residuos: prevención, reutilización, reciclaje, valorización energética y por último la eliminación, en orden de preferencia de mayor a menor (MMA, 2016).

El segundo es el principio preventivo, definido en la REP así:

Conjunto de acciones o medidas que se reflejan en cambios en los hábitos en el uso de insumos y materias primas utilizadas en procesos productivos, Diseño o en modificaciones en dichos procesos, así como en el consumo, destinadas a evitar la generación de residuos, la reducción en cantidad o la peligrosidad de los mismos (MMA, 2016, p.2).

El enfoque preventivo apunta a lograr cambios de hábitos por parte del sector productivo y los consumidores para evitar la generación de residuos y reducir su impacto. Explícitamente la ley incluye el Diseño de productos y procesos para lograr estos objetivos.

\section{Economía circular}

La economía circular se transforma globalmente en la aproximación preferida por parte de empresas, gobiernos y organizaciones para la reducción de impactos derivados de residuos. Esto ha sucedido gracias a la organización Ellen MacArthur Foundation (EMF). EMF propone pasar del modelo lineal extractivo actual de tomar-producir-desechar, a una economía circular que ofrece una visión positiva de economía en que los productos están diseñados para ser reutilizados, reparados o reciclados y los sistemas naturales se regeneran (EMF, 2020). 
El concepto de economía circular cuenta con múltiples antecedentes. Tradicionalmente en la economía han existido flujos lineales de materiales, desde la naturaleza hacia la economía y luego de vuelta hacia la naturaleza como residuos. Ante esto, se ha propuesto reemplazar flujos lineales por flujos circulares en que los residuos vuelven a ingresar a la economía como productos (McDonough \& Braungart, 2002). Se distinguen ciclos biológicos y ciclos técnicos para el manejo de materiales, según su naturaleza (McDonough \& Braungart, 2002).

Pearce y Turner elaboraron sobre economía circular hace más de tres décadas en su libro Economía de Recursos Naturales y Medio Ambiente. En el texto los autores explican las interacciones que existen entre la economía y el medio ambiente, las implicancias del crecimiento económico para las funciones del medio ambiente y la importancia de las leyes de la termodinámica para la economía (Pearce \& Turner, 1989). Estas leyes permiten comprender por qué cualquier cosa o recurso que usemos va a quedar en el medio ambiente, ante lo cual los autores proponen una economía circular en que los residuos se reciclan, tal como sucede en la naturaleza (Pearce \& Turner, 1989).

La primera ley de la termodinámica establece que la materia y la energía no pueden crearse ni destruirse, sólo transformarse. La segunda ley establece que en un sistema cerrado la entropía sólo aumentará; es decir, la materia y la energía tienden a disiparse, a la desorganización y al caos. Al ser una ley física esto se aplica a todo objeto físico, a toda materia y energía.

Trabajos previos ya habían destacado la importancia de las leyes de la termodinámica para la economía. Un trabajo señero es The Economics of the Coming Spaceship Earth, del economista Kenneth Boulding. También destaca la obra del matemático Nicholas Georgescu-Roegen The Entropy Law and the Economic Problem. Más recientemente se aborda la relevancia de las leyes de la termodinámica para la economía en el libro Ecological Economics, por Daly \& Farley (2011).

\section{Discusión y Conclusiones.}

\section{Importancia de las leyes de la termodinámica}

Las leyes de la termodinámica son leyes físicas. Como tales no es posible no cumplirlas, a diferencia de las leyes sociales. Los objetos físicos están, o estamos, todos sujetos a ellas.

Por esto, los materiales utilizados en el ciclo de vida de envases y embalajes no pueden crearse sino que siempre provienen de alguna fuente en nuestro sistema Tierra. Esto nos lleva a un problema de disponibilidad de recursos. De manera similar, los residuos sólidos, efluentes y emisiones generadas en el ciclo de vida de envases no pueden desaparecer o destruirse, sino que permanecerán también en el sistema. Esto nos lleva a un problema de residuos, en particular la contaminación de la biósfera.

En cuanto a la energía, si bien la Tierra es un sistema cerrado a la materia, ya que nada entra ni sale significativamente, es un sistema abierto a la energía. La gran cantidad de energía solar que entra al sistema Tierra cada día permite 
los procesos biológicos. Nosotros al utilizar la energía solar no generamos emisiones in situ, si bien en la producción de la tecnología e infraestructura necesarias para cosechar esta energía probablemente se generan emisiones debido al uso de combustibles fósiles. El flujo de energía solar que llega a la Tierra es gratis e ilimitado (al menos a escala humana). Eventualmente en un futuro podríamos utilizar sólo fuentes de energía solar y con esto lograr una economía realmente circular.

Al crear nuevos productos, las leyes de la termodinámica se expresan especialmente en la disponibilidad de recursos para producirlos y en la generación de residuos. Será necesario utilizar ecodiseño en los procesos de Diseño, con atención a la disponibilidad de recursos, a las capacidades del medio ambiente de procesar residuos biológicos, y del sistema industrial de procesar residuos técnicos. Las energías que utilicen deberán ser de origen solar y las tecnologías para captar y hacer disponibles estas energías para su uso deberán ser limpias.

\section{No basta con la REP}

Hoy un porcentaje menor de los residuos de envases y embalajes en Chile se reutilizan o reciclan, situación que cambiaría con la REP en pleno funcionamiento. La REP puede ayudar a solucionar en parte los problemas de residuos de envases post consumo, pero la ley no aborda las demás etapas del ciclo de vida de los productos prioritarios, de las cuales puede provenir un significativo impacto ambiental.

El ecodiseño mencionado en la ley, sin embargo, se enfoca en las distintas etapas del ciclo de vida y puede ayudar a minimizar impactos negativos en cada etapa. Esta herramienta permite reducir impactos, no solo del envase, sino también del producto envasado, los que pueden ser significativos. Por ejemplo, un LCA sobre el café en grano mostró que los principales impactos provienen de la producción del café y su preparación para el consumo; el envase y transporte son de menor importancia (Büsser \& Jungbluth, 2009).

Por otra parte, la REP se enfoca en productos prioritarios, pero no en los materiales que los constituyen. Paradójicamente, es precisamente por los materiales componentes de los productos prioritarios que éstos están afectos a la ley. En envases y embalajes se pueden encontrar materiales indeseables en una corriente de residuos. Por ejemplo, el PVc es un plástico conocido por su impacto ambiental y se utiliza en algunos envases. Este material será obligatorio gestionarlo cuando se encuentre en envases y embalajes. Sin embargo, el Pvc también se utiliza en otros productos (e.g. domésticos o de construcción) que no están afectos a la ley. Es importante cumplir la REP, sobre todo si consideramos el principio de jerarquía para minimizar impactos; pero ésta permite resolver sólo en parte los problemas ambientales de los productos prioritarios.

El concepto de valorización, presente en la ley, puede constituir un arma de doble filo. La REP habla de producir productos o energía a partir de los residuos (MMA, 2016). Producir energía se refiere a la valorización energética, o utilizar los residuos como combustible alternativo. Efectivamente existe energía incorporada en los materiales de envases (e.g. plásticos y papeles) que se puede recuperar mediante la incineración, para reemplazar otros 
combustibles. De hecho, es práctico hacerlo con envases de alto contenido energético y con envases conformados por múltiples materiales difíciles de separar o que contaminarían el reciclaje. Pero la valorización energética es contaminante, por lo cual se encuentra abajo en la estrategia jerarquizada para el manejo de residuos.

\section{Rol de la fase de Diseño en una economía circular}

En una economía circular de envases y embalajes se manejarían los materiales de los residuos en ciclos cerrados para crear productos con valor económico. Durante la fase de Diseño de un producto se toman decisiones que determinan su desempeño ambiental de ciclo de vida. Esto influye en la etapa de fin de vida, donde se enfoca la REP. Un Diseño apropiado puede facilitar su cumplimiento y uno inadecuado puede dificultarlo o impedirlo. El implementar ecodiseño durante la fase de Diseño de envases tiene gran potencial para minimizar sus impactos. Un adecuado Diseño de envase permite que sus residuos post consumo sean efectivamente reutilizables, compostables o reciclables; en síntesis, valorizables en una economía circular.

Para integrar el ecodiseño con propiedad en envases y embalajes, es necesario incorporar el conocimiento adecuado en los procesos de Diseño. Este conocimiento debe incluir información sobre el impacto ambiental de ciclo de vida del sistema producto a diseñar, idealmente LCA. En base a esta información se pueden identificar problemas ambientales, para luego planificar y diseñar soluciones de menor impacto con el ecodiseño. Luego se puede evaluar la solución y comparar con los impactos del producto anterior. producto nuevo podría generar mayor impacto que el anterior.

Si bien el ecodiseño tiene un foco en el producto físico, el Diseño para la comunicación en el sistema de envases y embalajes tiene roles importantes en una economía circular. Esta comunicación incluye tanto la cara frontal como posterior del envase primario, así como los embalajes usados. El comunicar los atributos del producto o su envase, cuando tienen un desempeño ambiental optimizado, es importante para informar al consumidor. Similarmente, comunicar al comprador si el producto o envase tienen sellos o certificaciones mediante la gráfica en los envases puede influenciar sus decisiones de compra.

Adicionalmente el envase puede contener información para el correcto manejo de sus residuos. Ésta puede incluir datos sobre materiales, instrucciones de preparación de residuos para su gestión, y cómo debe entregarse a un sistema de gestión para su valorización. Si el consumidor final no realiza las funciones que le corresponden entonces no se cierra un ciclo de economía circular. Para ayudar a que las cumpla, el Diseño para la comunicación es fundamental.

\section{Ventajas para las disciplinas de Diseño y rol potencial que pueden cumplir}

La REP constituye un aliciente para el desarrollo de las disciplinas de Diseño. Durante la fase de Diseño de un producto se toman decisiones sobre su creación, desde planificación y decisiones estratégicas hasta el Diseño de detalles. Algunas disciplinas de Diseño están tradicionalmente 
relacionadas con envases y embalajes, e.g. Diseño de envases, Diseño gráfico y comunicación visual, ingeniería y Diseño de productos, marketing y publicidad. Éstas pueden tener un rol importante para la efectividad de la REP y el cumplimiento de sus objetivos.

Por una parte, el ecodiseño emerge como una rama de desarrollo profesional y sofisticación de funciones para las disciplinas de Diseño relacionadas con envases y embalajes. Esto constituye una posible puesta al día o actualización para estas profesiones y sus ámbitos de trabajo. Se requerirá incorporar conocimiento adicional a los saberes de estos profesionales, tanto a nivel de profundización profesional como de formación de pregrado. Además, al ser requerido por ley y al no contar hoy con envases que puedan ser valorizados, serán necesarios nuevos Diseños y aumentará la demanda de los servicios de estos profesionales.

Por otra parte, los componentes del principio preventivo de la REP en su totalidad son accionables durante la fase de Diseño de productos y envases. Esto pone a las disciplinas de Diseño, que habitualmente trabajan en esta fase, en una posición privilegiada como instrumentos para el cumplimiento de la REP y el cuidado del medio ambiente.

Complementariamente, la industria deberá demandar más información ambiental sobre los productos y su ecodiseño a fin de cumplir la REP. Esta información habitualmente proviene de universidades, centros de investigación, científicos y consultores ambientales. Esto también debiera expandir y desarrollar el sector del conocimiento sobre aspectos ambientales del producto.

También debieran verse potenciados los rubros de Diseño de productos a partir de la reutilización y el reciclaje, es decir, bienes y servicios a partir de la valorización de residuos. Este tipo de productos son indispensables para lograr realmente una economía circular. Los materiales reciclados no tendrían demanda si no existieran productos manufacturados con estos materiales y la reutilización también requiere que existan productos que funcionen con este principio. Por lo tanto, deberán desarrollarse estas industrias y el Diseño relacionado con ellas.

Por último, si se observa el ciclo de vida del producto y especialmente la gestión de residuos, debiera fortalecerse este sector a través de los distintos actores que ejecutan funciones dentro de él. Esto atañe al desarrollo de áreas técnicas como el Diseño de procesos productivos y materiales, y también a los múltiples servicios que deben operar para el adecuado funcionamiento de sistemas de gestión de residuos.

\section{Nota del Autor}

Agradecimientos a la Escuela de Diseño de la Pontificia Universidad Católica de Chile, por proveer los recursos e infraestructura necesarias para la investigación sobre impacto ambiental y Diseño de productos. 


\section{Referencias}

Aylwin, M. \& Bortnick, D. (2017). La Ley $n^{\circ} 20.920$ y la era 'REP': un cambio de paradigma en el manejo de residuos en Chile. Actualidad Jurídica, 36, 315-348.

Büsser, S. \& Jungbluth, N. (2009). The role of flexible packaging in the life cycle of coffee and butter. International Journal of Life Cycle Assessment, 14(1), 8091. DOI 10.1007/S11367-008-0056-2

Cabeza, L. F., Rincón, L., Vilariño, V., Pérez, G. \& Castell, A. (2014). Life cycle assessment (LCA) and life cycle energy analysis (LCEA) of buildings and the building sector: A review. Renewable and Sustainable Energy Reviews, 29, 394-416. https://doi.org/10.1016/j. rser.2013.08.037

Ciravegna, E. (2017). Diseño de packaging. Una aproximación sistémica a un artefacto complejo. RChD: creación y pensamiento, 2(3), 1-17. https://doi. org/10.5354/0719-837X.2017.47825

Daly, H. E. \& Farley, J. (2011). Ecological economics principles and applications. ISLAND PRESS.

Dormer, A., Finn, D. P., Ward, P. \& Cullen, J. (2013). Carbon footprint analysis in plastics manufacturing. Journal of Cleaner Production, 51, 133-141.

Ellen MacArthur Foundation. (2020). Financing the circular economy: Capturing the opportunity. https://www. ellenmacarthurfoundation.org/assets/downloads/ Financing-the-circular-economy.pdf

Huerta, O. (2014). Integrating environmentally responsible design with life cycle assessment in product and process development for sustainability (No. 3619907) 1-238 [Tesis de doctorado, Arizona State University]. ProQuest Dissertations and Theses.

International Organization for Standardization. (2006). Environmental management - Life cycle assessment Requirements and guidelines. Iso.

Karlsson, R. \& Luttropp, C. (2006). EcoDesign: What's happening? An overview of the subject area of EcoDesign and of the papers in this special issue. Journal of Cleaner Production, 14(15-16), 1291-1298. https://doi.org/10.1016/j.jclepro.2005.11.010

Kiser, B. (2016). Circular economy: getting the circulation going. Nature, 531, 443-446.
McDonough, W. \& Braungart, M. (2002). Cradle to cradle: remaking the way we make things. NORTH POINT PRESS. Ministerio del Medio Ambiente de Chile. (2016, Junio 1). Ley $\mathrm{N}^{\circ}$ 20.920 Marco para la gestión de residuos, la responsabilidad extendida del productor y fomento al reciclaje. Diario Oficial de la República de Chile.

Ministerio del Medio Ambiente de Chile. (2020). Economía Circular. https://mma.gob.cl/economia-circular/.

Pearce, D. W. \& Turner, R. K. (1990). Economics of natural resources and the environment. JOHNS HOPKINS UNIVERSITY PRESS.

Sterrenberg, L., Böttcher, H. \& de Hoo, S. D. (1997, July). Ecodesign: A successful TA-project, a short retrospective. TATuP - Journal for Technology Assessment in Theory and Practice, Vol. 6 (2), 43-46. https://tatup.de/index.php/tatup/article/view/4504 DOI: https://doi.org/10.14512/tatup.6.2.43

Tischner, U., Schmincke, E., Rubik, F., \& Prosler, M. (2000). How to do Ecodesign? VERLAG FORM PRAXIS.

Wenzel, H., Hauschild, M. \& Alting, L. (1997). Environmental assessment of products, Vol. 1. CHAPMAN \& HALL.

White, P., St. Pierre, L. \& Belletire, S. (2013). Okala Practitioner: Integrating ecological design. OKALA TEAM. 